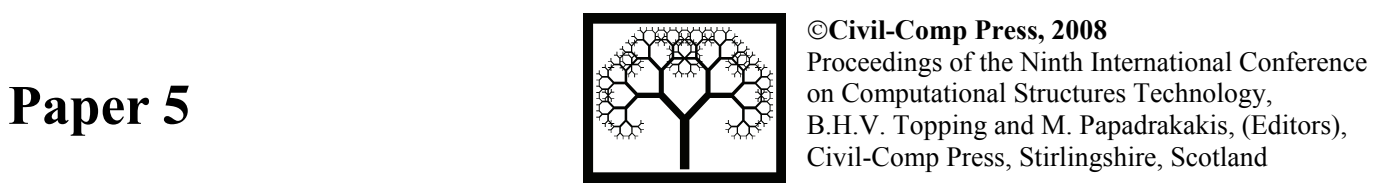

\title{
A Layerwise Mixed Least-Squares Finite Element Model for Static Analysis of Multilayered Composite Plates
}

\author{
F. Moleiro ${ }^{1}$, C.M. Mota Soares ${ }^{1}$, C.A. Mota Soares ${ }^{1}$ and J.N. Reddy $^{2}$ \\ ${ }^{1}$ Department of Mechanical Engineering, IDMEC/IST - \\ Instituto Superior Técnico, Technical University of Lisbon, Portugal \\ ${ }^{2}$ Department of Mechanical Engineering \\ Texas A\&M University, United States of America
}

\begin{abstract}
A layerwise finite element model is developed in a mixed least-squares formulation for static analysis of multilayered composite plates. The model assumes a layerwise variable description of displacements, transverse stresses and in-plane strains, taken as independent variables. The mixed formulation allows to completely and a priori fulfil the known $C_{z}^{0}$ requirements, which refer to the zig-zag form of displacements in the thickness direction and the interlaminar continuity of transverse stresses, due to compatibility and equilibrium reasons, respectively. This contrasts with layerwise displacement-based models that usually cannot a priori account for the interlaminar continuity of transverse stresses. In addition, the benefit of mixed least-squares formulation, as opposed to mixed weak form models, is that it leads to a variational unconstrained minimization problem, where the finite element approximating spaces can be chosen independently. Numerical examples are shown to assess the layerwise mixed least-squares model predictive capabilities compared to three-dimensional elasticity solutions and also other finite element results available in literature. Most notably, the present model is able to achieve accurate results in very good agreement with three-dimensional solutions and is shown to be insensitive to shear-locking.
\end{abstract}

Keywords: layerwise theory, mixed formulation, least-squares formulation, finite element model, multilayered composite plate.

\section{Introduction}

As multilayered composite structures are increasingly evolving from secondary to primary structural components, the role of structural analysis is enlarged to reach a highly accurate assessment of the structure response. In overview, the main available theories for multilayered composite plates and shells are axiomatic type approaches, which rely on certain assumptions concerning the thickness $z$-expansion for the chosen unknown variables. A historical view and evaluation of the most important 
theories is presented in the review articles [1-3] and in Reddy [4, 5]. The theories differ in equivalent single-layer or layerwise variable descriptions as well as in the chosen unknown variables, whether displacement, stress or mixed formulations. In equivalent single-layer variable descriptions the variables are introduced for the whole plate or shell, whereas in layerwise variable descriptions each layer is seen as an independent plate or shell, so the number of independent variables is dependent on the number of layers. Typically, variational principles have been established in literature to derive governing equations consistent with the chosen formulations. The widespread displacement formulations relate to the well-known principle of virtual displacement and the alternative mixed formulations derive from the Hu-Washizu or the Hellinger-Reissner variational principles $[6,7]$.

As demonstrated by early three-dimensional elasticity solutions by Pagano and Hatfield $[8,9]$, multilayered composite structures may exhibit complicating effects introduced by anisotropic behaviour, such as high transverse deformability, zig-zag effects and interlaminar continuity. Actually, multilayered composite structures may show high in-plane anisotropy due to different mechanical-physical properties in different in-plane directions. Consequently, these structures can display higher transverse flexibility with respect to in-plane deformability when compared to the isotropic single-layer structures. In other words, multilayered composite structures are high transversely deformable structures. Furthermore, multilayered constructions are also transversely anisotropic structures given the different mechanical-physical properties in the thickness direction. As a result, the displacements may exhibit discontinuous derivatives in correspondence to each layer interface. This is known as the zig-zag (ZZ) form of the displacements in the thickness direction. In addition, for equilibrium reasons, the transverse stresses must be continuous at each layer interface, which is known in literature as interlaminar continuity (IC). Ultimately, both displacements and transverse stresses must be $C^{0}$ continuous functions in the thickness $z$-direction, due to compatibility and equilibrium reasons, respectively. Altogether, ZZ and IC are usually referred to as $C_{z}^{0}$ requirements $[10,11]$. The fulfilment of $C_{z}^{0}$ requirements is crucial in the development of any theory suitable for multilayered structures.

Classical finite element models originally developed for single-layer isotropic structures were early on extended to multilayered composite plates and shells, as equivalent single-layer models (ESLMs). Namely, the classical lamination theory (CLT) and first-order shear deformation theory (FSDT) are known to simply provide a reasonable description of the global response of multilayered structures, without accounting for $C_{z}^{0}$ requirements. Improvements through partial fulfilment of $C_{z}^{0}$ requirements were later on achieved by higher-order shear deformation theories (HSDTs). Nevertheless, because of the intrinsic material couplings between the transverse normal and in-plane stress components, ESLMs experience difficulties in extending zig-zag forms to the transverse displacement or in accounting for the interlaminar continuity of the transverse normal stress. Hence, ESLMs are rather deficient in analysing very thick multilayered structures, in which the transverse normal stress plays a determinant role. In this respect, layerwise models (LWMs), in which two-dimensional approximations are introduced at a layer level, are much 
better suited to attain an acceptable level of accuracy compared to three-dimensional solutions. However, most available LWMs do not a priori and completely fulfil $C_{z}^{0}$ requirements. The typical displacement-based LWMs cannot a priori account for the interlaminar continuity of transverse stresses, which instead are usually evaluated a posteriori by integration of the three-dimensional equilibrium equations.

The convenience of assuming as independent variables equally displacements and transverse stresses intuitively appeared to Reissner $[12,13]$, who proposed a mixed formulation as a tool to variationally derive governing equilibrium and constitutive equations in terms of these independent variables. Some ESLMs made use of this mixed formulation, but for an accurate description of local response of multilayered composite structures, LWMs are required. Specifically, Carrera developed LWMs based upon Reissner's mixed variational theorem that completely and a priori fulfil $C_{z}^{0}$ requirements with very successful results [14-18]. Later on, Lage et al. [19, 20] extended the layerwise mixed model by Carrera to include piezoelectric/magnetic plates achieving good results as well.

Finite element models are normally based on weak formulations, whether ESLMs or LWMs, with displacement-based or mixed formulations. One of most mentioned issues in finite element modelling is shear-locking problems, which refer to the computational difficulties encountered when modelling thin plates or shells. Shearlocking is generally handled by numerical integration techniques or by using higherorder elements that experience relatively less locking but at the expense of a slower convergence. An additional issue in mixed weak formulations is that to be reliable the finite element approximation spaces must satisfy a so called inf-sup condition. The alternative to weak formulations is weighted residual formulations. Among them, least-squares formulations are unique in the basic idea of minimizing the error introduced in the approximation of the governing equations. In fact, the benefit of least-squares variational principle combined with a mixed formulation is that it leads to a variational unconstrained minimization problem, where the finite element approximating spaces can be chosen independently. Hence, stability requirements as the inf-sup condition never arise. Moreover, mixed least-squares formulations yield symmetric positive-definite stiffness matrices, contrary to mixed weak formulations. Earlier works on mixed least-squares formulations show promising theoretical and computational advantages. Specifically, Pontaza and Reddy [21] developed a mixed least-squares model for bending of single-layer isotropic plates, using the classical plate theory (CPT) and FSDT. Pontaza [22] also demonstrated the merit of leastsquares finite element models applied to both solid and fluid mechanics. The present authors' previous works extended Pontaza and Reddy mixed least-squares FSDT finite element model to static and free vibration analysis of multilayered composite plates $[23,24]$. Most notably, these preceding works show that mixed least-squares models using high-order $C^{0}$ basis functions in the two-dimensional approximations with full integration are insensitive to shear-locking. In fact, Pontaza and Reddy [21, 22] demonstrated the exponential decay of the least-squares functional for increasing $p$-order expansion of the two-dimensional approximations.

From the overall discussion, it emerges that (a) LWMs are required to accurately describe multilayered composite structures, $(b)$ mixed formulations are indeed useful 
to completely and a priori fulfil $C_{z}^{0}$ requirements and $(c)$ the benefits of mixed leastsquares formulations motivates the present development of a layerwise mixed leastsquares model for analysis of multilayered composite plates.

This paper is organised as follows. Section 2 is devoted to the description of the present model: the chosen layerwise variable description in a mixed formulation is presented, the system of consistent governing equations is established, the leastsquares formulation is introduced and in the end, the layerwise mixed least-squares model is shown. Section 3 presents numerical examples to assess the present model predicted capabilities compared to three-dimensional elasticity solutions and other finite element results available in literature. The last Section 4 summarizes the main conclusions.

\section{Layerwise Mixed Least-Squares Model}

Consider the multilayered plate of total thickness $h$ and rectangular planar geometry $\Omega$ composed of $N$ orthotropic layers, as shown in Figure 1. A rectangular coordinate system $(x, y, z)$ is used with the $z$-axis taken positive upward from the midplane along the thickness direction. The superscript $k$ is assigned to $k$-layer specifics, while the subscripts $b$ and $t$ designate the layer bottom and top surfaces, respectively.
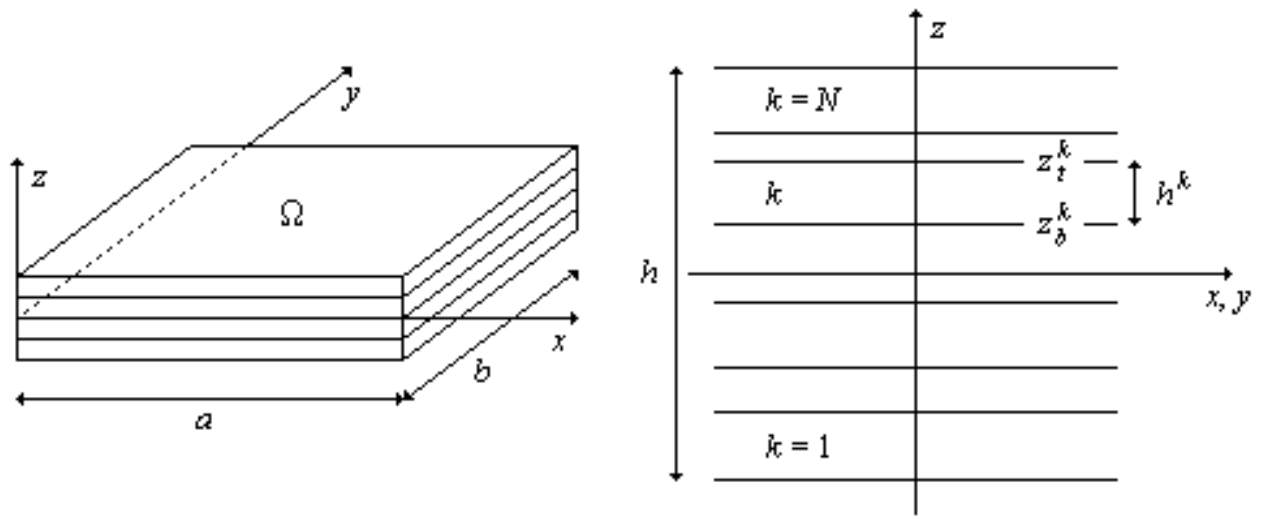

Figure 1: Geometry and notation of a multilayered plate.

\subsection{Layerwise Variable Description}

To completely and a priori fulfil $C_{z}^{0}$ requirements, a layerwise variable description of both displacements $\left(u^{k}, v^{k}, w^{k}\right)$ and transverse stresses $\left(\sigma_{x z}^{k}, \sigma_{y z}^{k}, \sigma_{z z}^{k}\right)$ needs to be assumed. Additionally, the chosen mixed formulation assumes a layerwise variable description of in-plane strains $\left(\varepsilon_{x x}^{k}, \varepsilon_{y y}^{k}, \gamma_{x y}^{k}\right)$, merely to ensure a first-order system of consistent governing equations in the end. This is considered in the best interest of least-squares formulation as it will be explained later on. Accordingly, the assumed expansions in the thickness direction of each $k$-layer, for all independent variables, 
are the following:

$$
\begin{aligned}
& u^{k}(x, y, z)=\sum_{s=1}^{m_{u}} u_{s}^{k}(x, y) \phi_{u s}{ }^{k}(z), \quad \sigma_{x z}{ }^{k}(x, y, z)=\sum_{s=1}^{m_{\sigma_{x z}}} \sigma_{x z s}{ }^{k}(x, y) \phi_{\sigma_{x z} s}{ }^{k}(z) \\
& v^{k}(x, y, z)=\sum_{s=1}^{m_{v}} v_{s}^{k}(x, y) \phi_{v s}{ }^{k}(z), \quad \sigma_{y z}{ }^{k}(x, y, z)=\sum_{s=1}^{m_{\sigma_{y z}}} \sigma_{y z s}{ }^{k}(x, y) \phi_{\sigma_{y z}}{ }^{k}(z) \\
& w^{k}(x, y, z)=\sum_{s=1}^{m_{w}} w_{s}^{k}(x, y) \phi_{w s}{ }^{k}(z), \quad \sigma_{z z}{ }^{k}(x, y, z)=\sum_{s=1}^{m_{\sigma_{z z}}} \sigma_{z z s}{ }^{k}(x, y) \phi_{\sigma_{z z} s}{ }^{k}(z) \\
& \varepsilon_{x x}{ }^{k}(x, y, z)=\sum_{s=1}^{m_{\varepsilon_{x x}}} \varepsilon_{x x s}{ }^{k}(x, y) \phi_{\varepsilon_{x x} s}{ }^{k}(z), \quad \varepsilon_{y y}{ }^{k}(x, y, z)=\sum_{s=1}^{m_{\varepsilon_{y y}}} \varepsilon_{y y_{s}}{ }^{k}(x, y) \phi_{\varepsilon_{y y} s}{ }^{k}(z) \\
& \gamma_{x y}{ }^{k}(x, y, z)=\sum_{s=1}^{m_{\gamma_{x y}}} \gamma_{x y_{s}}{ }^{k}(x, y) \phi_{\gamma_{x y}}{ }^{k}(z)
\end{aligned}
$$

The functions $\phi_{u_{s}}{ }^{k}(z), \ldots, \phi_{\gamma_{x y_{s}}}{ }^{k}(z)$ are one-dimensional $C^{0}$ nodal basis functions in the thickness direction, which are defined only within the $k$-layer. The number of nodes through the layer thickness, $m_{u}, \ldots, m_{\gamma_{x y}}$ define the expansion $p$-order in the $z$-direction of the $k$-layer, as $p=m-1$. Hence, the functions $u_{s}^{k}(x, y), \ldots, \gamma_{x y_{s}}{ }^{k}(x, y)$ denote the variables nodal values at the $s$-plane of the $k$-layer. Details on the adopted $C^{0}$ basis functions are given later in Subsection 2.4.

\subsubsection{Interlaminar Compatibility and Equilibrium Conditions}

In view of the adopted layerwise mixed formulation $C_{z}^{0}$ requirements can easily be completely and a priori fulfilled just by assembling the layers. See Carrera $[14,18]$ for details on assembly from layer to multilayer. In other words, the top and bottom surfaces of adjacent layers must agree in both displacements and transverse stresses, as stated by $C_{z}^{0}$ requirements, but also in the in-plane strains (which is legitimate), given the chosen mixed formulation. Altogether, the interlaminar conditions are:

$$
\begin{gathered}
u_{t}^{k}=u_{b}^{k+1}, v_{t}^{k}=v_{b}^{k+1}, w_{t}^{k}=w_{b}^{k+1}, \quad \text { for } k=1, \ldots, N-1 \\
\sigma_{x z t}{ }^{k}=\sigma_{x z b}{ }^{k+1}, \sigma_{y z t}{ }^{k}=\sigma_{y z b}{ }^{k+1}, \sigma_{z z t}{ }^{k}=\sigma_{z z b}{ }^{k+1}, \quad \text { for } k=1, \ldots, N-1 \\
\varepsilon_{x x t}{ }^{k}=\varepsilon_{x x b}{ }^{k+1}, \varepsilon_{y y_{t}}{ }^{k}=\varepsilon_{y y_{b}}{ }^{k+1}, \gamma_{x y_{t}}{ }^{k}=\gamma_{x y_{b}}{ }^{k+1}, \quad \text { for } k=1, \ldots, N-1
\end{gathered}
$$

\subsection{Governing Equations for the Layer}

The fundamental equations for linear static analysis of each $k$-layer, which is seen as an individual plate, are the equilibrium equations, the constitutive equations and the strain-displacement equations, consistent with three-dimensional linear elasticity, as 
follows:

- Equilibrium Equations

$$
\begin{aligned}
& \frac{\partial \sigma_{x x}{ }^{k}}{\partial x}+\frac{\partial \sigma_{x y}{ }^{k}}{\partial y}+\frac{\partial \sigma_{x z}{ }^{k}}{\partial z}+f_{x}{ }^{k}=0 \\
& \frac{\partial \sigma_{x y}{ }^{k}}{\partial x}+\frac{\partial \sigma_{y y}{ }^{k}}{\partial y}+\frac{\partial \sigma_{y z}{ }^{k}}{\partial z}+f_{y}{ }^{k}=0 \\
& \frac{\partial \sigma_{x z}{ }^{k}}{\partial x}+\frac{\partial \sigma_{y z}{ }^{k}}{\partial y}+\frac{\partial \sigma_{z z}{ }^{k}}{\partial z}+f_{z}{ }^{k}=0
\end{aligned}
$$

- Constitutive Equations (in a matrix form)

$$
\begin{aligned}
& \left\{\begin{array}{l}
\sigma_{x x}{ }^{k} \\
\sigma_{y y}{ }^{k} \\
\sigma_{x y}{ }^{k}
\end{array}\right\}=\left[\begin{array}{ccc}
\bar{C}_{11}{ }^{k} & \bar{C}_{12}{ }^{k} & \bar{C}_{16}{ }^{k} \\
\bar{C}_{12} & \bar{C}_{22}{ }^{k} & \bar{C}_{26}{ }^{k} \\
\bar{C}_{16}{ }^{k} & \bar{C}_{26}{ }^{k} & \bar{C}_{66}{ }^{k}
\end{array}\right]\left\{\begin{array}{c}
\varepsilon_{x x}{ }^{k} \\
\varepsilon_{y y}{ }^{k} \\
\gamma_{x y}{ }^{k}
\end{array}\right\}+\left[\begin{array}{ccc}
0 & 0 & \bar{C}_{13}{ }^{k} \\
0 & 0 & \bar{C}_{23}{ }^{k} \\
0 & 0 & \bar{C}_{36}{ }^{k}
\end{array}\right]\left\{\begin{array}{l}
\gamma_{x z}{ }^{k} \\
\gamma_{y z}{ }^{k} \\
\varepsilon_{z z}{ }^{k}
\end{array}\right\} \\
& \left\{\begin{array}{l}
\sigma_{x z}{ }^{k} \\
\sigma_{y z}{ }^{k} \\
\sigma_{z z}{ }^{k}
\end{array}\right\}=\left[\begin{array}{ccc}
0 & 0 & 0 \\
0 & 0 & 0 \\
\bar{C}_{13}{ }^{k} & \bar{C}_{23}{ }^{k} & \bar{C}_{36}{ }_{36}
\end{array}\right]\left\{\begin{array}{l}
\varepsilon_{x x}{ }^{k} \\
\varepsilon_{y y}{ }^{k} \\
\gamma_{x y}{ }^{k}
\end{array}\right\}+\left[\begin{array}{ccc}
\bar{C}_{55}{ }^{k} & \bar{C}_{45}{ }^{k} & 0 \\
\bar{C}_{45}{ }^{k} & \bar{C}_{44}{ }^{k} & 0 \\
0 & 0 & \bar{C}_{33}{ }^{k}
\end{array}\right]\left\{\begin{array}{l}
\gamma_{x z}{ }^{k} \\
\gamma_{y z}{ }^{k} \\
\varepsilon_{z z}{ }^{k}
\end{array}\right\}
\end{aligned}
$$

- Strain-Displacement Equations

$$
\begin{gathered}
\varepsilon_{x x}{ }^{k}=\frac{\partial u^{k}}{\partial x}, \varepsilon_{y y}{ }^{k}=\frac{\partial v^{k}}{\partial y}, \gamma_{x y}{ }^{k}=\frac{\partial u^{k}}{\partial y}+\frac{\partial v^{k}}{\partial x} \\
\gamma_{x z}{ }^{k}=\frac{\partial u^{k}}{\partial z}+\frac{\partial w^{k}}{\partial x}, \gamma_{y z}{ }^{k}=\frac{\partial v^{k}}{\partial z}+\frac{\partial w^{k}}{\partial y}, \varepsilon_{z z}{ }^{k}=\frac{\partial w^{k}}{\partial z}
\end{gathered}
$$

These equations are written in reference to the multilayer plate coordinate system $(x, y, z)$ shown in Figure 1. Hence, $\bar{C}^{k}$ represent the transformed material stiffness coefficients of the (orthotropic) $k$-layer referred to the $(x, y, z)$ coordinate system. In addition, $f^{k}$ denotes the body force components per unit volume. See Reddy [4] for more details.

The key to derive a system of governing equations consistent with the partial mixed formulation chosen, resides in a convenient manipulation of the constitutive equations. The purpose is to write the in-plane stresses and transverse strains only as function of chosen independent variables, namely, the transverse stresses and the inplane strains. The matrix form used in the above constitutive equations with in-plane and transverse variables written separately, rather reveals the manipulations needed. Ultimately, the rearranged constitutive equations take the following form: 


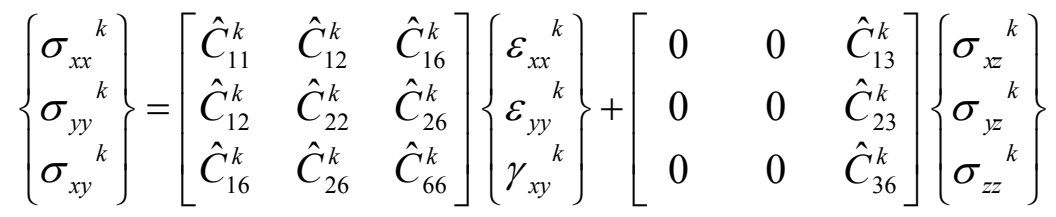

$$
\begin{aligned}
& \left\{\begin{array}{l}
\gamma_{x z}{ }^{k} \\
\gamma_{y z}{ }^{k} \\
\varepsilon_{z z}{ }^{k}
\end{array}\right\}=\left[\begin{array}{ccc}
0 & 0 & 0 \\
0 & 0 & 0 \\
-\hat{C}_{13}{ }^{k} & -\hat{C}_{23}{ }^{k} & -\hat{C}_{36}{ }_{36}
\end{array}\right]\left\{\begin{array}{l}
\varepsilon_{x x}{ }^{k} \\
\varepsilon_{y y}{ }^{k} \\
\gamma_{x y}{ }^{k}
\end{array}\right\}+\left[\begin{array}{ccc}
\hat{C}_{55} & \hat{C}_{45}{ }^{k} & 0 \\
\hat{C}_{45} & \hat{C}_{44}{ }^{k} & 0 \\
0 & 0 & \hat{C}_{33}{ }^{k}
\end{array}\right]\left\{\begin{array}{l}
\sigma_{x z}{ }^{k}{ }^{k} \\
\sigma_{y z}{ }^{k} \\
\sigma_{z z}{ }^{k}
\end{array}\right\}
\end{aligned}
$$

where,

$$
\begin{gathered}
\hat{C}_{i j}^{k}=\bar{C}_{i j}^{k}-\frac{\bar{C}_{i 3}^{k} \bar{C}_{j 3}^{k}}{\bar{C}_{33}^{k}}, \hat{C}_{i 3}^{k}=\frac{\bar{C}_{i 3}^{k}}{\bar{C}_{33}^{k}}, \hat{C}_{33}^{k}=\frac{1}{\bar{C}_{33}^{k}}, \quad i, j=1,2,6 \\
\hat{C}_{i i}^{k}=\frac{\bar{C}_{j j}^{k}}{\Delta}, \hat{C}_{i j}^{k}=\frac{-\bar{C}_{i j}^{k}}{\Delta}, \Delta=\bar{C}_{55}^{k} \bar{C}_{44}^{k}-\bar{C}_{45}^{k^{2}}, \quad i, j(i \neq j)=4,5
\end{gathered}
$$

The governing equations for linear static analysis of the $k$-layer, consistent with the chosen mixed formulation, can now be established. The equilibrium equations given in Equation (3) are rewritten using Equation (6a) in order to give equilibrium equations solely as function of transverse stresses and in-plane strains, which are independent variables of the mixed formulation. Hence, the differential equilibrium equations of each $k$-layer are considered as follows:

$$
\begin{gathered}
\hat{C}_{11}^{k} \frac{\partial \varepsilon_{x x}{ }^{k}}{\partial x}+\hat{C}_{12}^{k} \frac{\partial \varepsilon_{y y}{ }^{k}}{\partial x}+\hat{C}_{16}^{k} \frac{\partial \gamma_{x y}{ }^{k}}{\partial x}+\hat{C}_{13}^{k} \frac{\partial \sigma_{z z}{ }^{k}}{\partial x}+\hat{C}_{16}^{k} \frac{\partial \varepsilon_{x x}{ }^{k}}{\partial y}+ \\
+\hat{C}_{26}^{k} \frac{\partial \varepsilon_{y y}{ }^{k}}{\partial y}+\hat{C}_{66}^{k} \frac{\partial \gamma_{x y}{ }^{k}}{\partial y}+\hat{C}_{36}^{k} \frac{\partial \sigma_{z z}{ }^{k}}{\partial y}+\frac{\partial \sigma_{x z}{ }^{k}}{\partial z}+f_{x}{ }^{k}=0 \\
\hat{C}_{16}{ }^{k} \frac{\partial \varepsilon_{x x}{ }^{k}}{\partial x}+\hat{C}_{26}{ }^{k} \frac{\partial \varepsilon_{y y}{ }^{k}}{\partial x}+\hat{C}_{66}^{k} \frac{\partial \gamma_{x y}{ }^{k}}{\partial x}+\hat{C}_{36}{ }^{k} \frac{\partial \sigma_{z z}{ }^{k}}{\partial x}+\hat{C}_{12}{ }^{k} \frac{\partial \varepsilon_{x x}{ }^{k}}{\partial y}+ \\
+\hat{C}_{22}{ }^{k} \frac{\partial \varepsilon_{y y}{ }^{k}}{\partial y}+\hat{C}_{26}{ }^{k} \frac{\partial \gamma_{x y}{ }^{k}}{\partial y}+\hat{C}_{23}{ }^{k} \frac{\partial \sigma_{z z}{ }^{k}}{\partial y}+\frac{\partial \sigma_{y z}{ }^{k}}{\partial z}+f_{y}{ }^{k}=0 \\
\frac{\partial \sigma_{x z}{ }^{k}}{\partial x}+\frac{\partial \sigma_{y z}{ }^{k}}{\partial y}+\frac{\partial \sigma_{z z}{ }^{k}}{\partial z}+f_{z}{ }^{k}=0
\end{gathered}
$$

Furthermore, in view of the partial mixed formulation chosen, these equilibrium equations must be accompanied by partial constitutive equations and partial straindisplacement equations. Since transverse stresses and in-plane strains are chosen independent variables, the partial constitutive equations for transverse stresses given in Equation ( $4 b)$ and the partial strain-displacement equations for in-plane strains given in Equation ( $5 a$ ) must be considered. Nonetheless, Equation ( $4 b)$ needs to be rewritten using Equation $(5 b)$ in order to be in terms of independent variables only. 
Explicitly, these accompanying governing equations are:

$$
\begin{aligned}
& \sigma_{x z}{ }^{k}-\bar{C}_{55}^{k} \frac{\partial u^{k}}{\partial z}-\bar{C}_{55}^{k} \frac{\partial w^{k}}{\partial x}-\bar{C}_{45}^{k} \frac{\partial v^{k}}{\partial z}-\bar{C}_{45}^{k} \frac{\partial w^{k}}{\partial y}=0 \\
& \sigma_{y z}^{k}-\bar{C}_{45}^{k} \frac{\partial u^{k}}{\partial z}-\bar{C}_{45}^{k} \frac{\partial w^{k}}{\partial x}-\bar{C}_{44}^{k} \frac{\partial v^{k}}{\partial z}-\bar{C}_{44}^{k} \frac{\partial w^{k}}{\partial y}=0 \\
& \sigma_{z z}{ }^{k}-\bar{C}_{13}{ }^{k} \varepsilon_{x x}{ }^{k}-\bar{C}_{23}{ }^{k} \varepsilon_{y y}{ }^{k}-\bar{C}_{36}{ }^{k} \gamma_{x y}{ }^{k}-\bar{C}_{33}{ }^{k} \frac{\partial w^{k}}{\partial z}=0 \\
& \varepsilon_{x x}{ }^{k}-\frac{\partial u^{k}}{\partial x}=0, \varepsilon_{y y}{ }^{k}-\frac{\partial v^{k}}{\partial y}=0, \gamma_{x y}{ }^{k}-\frac{\partial u^{k}}{\partial y}-\frac{\partial v^{k}}{\partial x}=0
\end{aligned}
$$

Therefore, the complete system of consistent governing equations for linear static analysis of the $k$-layer, given by Equations (8), (9) and (10), consists of equilibrium equations, partial constitutive equations and partial strain-displacement equations, written in terms of all independent variables of the mixed formulation, namely, the displacements, transverse stresses and in-plane strains.

\subsection{Least-Squares Formulation}

From a practical standpoint, it is best to develop least-squares finite element models that allow the use of $C^{0}$ basis functions in the two-dimensional approximations, in order to reduce the higher regularity requirements common in weighted residual formulations. Therefore, whenever necessary the governing equations are preferably transformed into an equivalent first-order system, which requires the introduction of additional independent variables, leading to mixed least-squares formulations. This is the reason for assuming a layerwise variable description of in-plane strains, which are merely intended to ensure first-order equilibrium equations.

The least-squares functional is in general developed by measuring the norms of the residuals of the governing equations. Specifically, for the layerwise mixed model described, the least-squares functional is derived by the sum of the squared residuals of each governing equation for the $k$-layer, and taking into account the contribution of all layers, as follows:

$$
\begin{aligned}
J=\frac{1}{2} \sum_{k=1}^{N} \int_{\Omega} \int_{h^{k}}\left\{\left(\hat{C}_{11}^{k} \frac{\partial \varepsilon_{x x}{ }^{k}}{\partial x}+\hat{C}_{12}{ }^{k} \frac{\partial \varepsilon_{y y}{ }^{k}}{\partial x}+\hat{C}_{16}{ }^{k} \frac{\partial \gamma_{x y}{ }^{k}}{\partial x}+\hat{C}_{13}^{k} \frac{\partial \sigma_{z z}{ }^{k}}{\partial x}+\hat{C}_{16}{ }^{k} \frac{\partial \varepsilon_{x x}{ }^{k}}{\partial y}+\right.\right. \\
\left.+\hat{C}_{26}^{k} \frac{\partial \varepsilon_{y y}{ }^{k}}{\partial y}+\hat{C}_{66}^{k} \frac{\partial \gamma_{x y}{ }^{k}}{\partial y}+\hat{C}_{36}^{k} \frac{\partial \sigma_{z z}{ }^{k}}{\partial y}+\frac{\partial \sigma_{x z}{ }^{k}}{\partial z}+f_{x}{ }^{k}\right)^{2}+ \\
+\left(\hat{C}_{16}^{k} \frac{\partial \varepsilon_{x x}{ }^{k}}{\partial x}+\hat{C}_{26}^{k} \frac{\partial \varepsilon_{y y}{ }^{k}}{\partial x}+\hat{C}_{66}^{k} \frac{\partial \gamma_{x y}{ }^{k}}{\partial x}+\hat{C}_{36}^{k} \frac{\partial \sigma_{z z}{ }^{k}}{\partial x}+\hat{C}_{12}^{k} \frac{\partial \varepsilon_{x x}{ }^{k}}{\partial y}+\right.
\end{aligned}
$$




$$
\begin{gathered}
\left.+\hat{C}_{22}{ }^{k} \frac{\partial \varepsilon_{y y}{ }^{k}}{\partial y}+\hat{C}_{26}{ }^{k} \frac{\partial \gamma_{x y}{ }^{k}}{\partial y}+\hat{C}_{23}^{k} \frac{\partial \sigma_{z z}{ }^{k}}{\partial y}+\frac{\partial \sigma_{y z}{ }^{k}}{\partial z}+f_{y}{ }^{k}\right)^{2}+ \\
+\left(\frac{\partial \sigma_{x z}{ }^{k}}{\partial x}+\frac{\partial \sigma_{y z}{ }^{k}}{\partial y}+\frac{\partial \sigma_{z z}{ }^{k}}{\partial z}+f_{z}{ }^{k}\right)^{2}+ \\
+\left(\sigma_{x z}{ }^{k}-\bar{C}_{55}{ }^{k} \frac{\partial u^{k}}{\partial z}-\bar{C}_{55}{ }^{k} \frac{\partial w^{k}}{\partial x}-\bar{C}_{45}{ }^{k} \frac{\partial v^{k}}{\partial z}-\bar{C}_{45}{ }^{k} \frac{\partial w^{k}}{\partial y}\right)^{2}+ \\
+\left(\sigma_{y z}{ }^{k}-\bar{C}_{45}{ }^{k} \frac{\partial u^{k}}{\partial z}-\bar{C}_{45}{ }^{k} \frac{\partial w^{k}}{\partial x}-\bar{C}_{44}{ }^{k} \frac{\partial v^{k}}{\partial z}-\bar{C}_{44}{ }^{k} \frac{\partial w^{k}}{\partial y}\right)^{2}+ \\
+\left(\sigma_{z z}{ }^{k}-\bar{C}_{13}{ }^{k} \varepsilon_{x x}{ }^{k}-\bar{C}_{23}{ }^{k} \varepsilon_{y y}{ }^{k}-\bar{C}_{36}{ }^{k} \gamma_{x y}{ }^{k}-\bar{C}_{33}{ }^{k} \frac{\partial w^{k}}{\partial z}\right)^{2}+ \\
\left.+\left(\varepsilon_{x x}{ }^{k}-\frac{\partial u^{k}}{\partial x}\right)^{2}+\left(\varepsilon_{y y}{ }^{k}-\frac{\partial v^{k}}{\partial y}\right)^{2}+\left(\gamma_{x y}{ }^{k}-\frac{\partial u^{k}}{\partial y}-\frac{\partial v^{k}}{\partial x}\right)^{2}\right\} d z d \Omega
\end{gathered}
$$

The least-squares variational principle simply states that the desired solution for the independent variables, (within an admissible space that satisfies the boundary conditions), is the one that minimizes the least-squares functional. Hence, the mixed least-squares formulation leads to a variational unconstrained minimization problem. Specifically, the Euler-Lagrange equation is derived and conveniently rearranged so that terms associated to the variation of each independent variable are combined. This procedure is quite straightforward and extensive so it is not explicitly included.

In general, arbitrary weights may be assigned to each of the residuals in the leastsquares functional. In fact, the implemented layerwise mixed least-squares model uses weights, chosen to non-dimensionalize the equations, in the interest of equally minimizing all residuals.

\subsection{Finite Element Model}

The subsequent finite element model is developed from the derived Euler-Lagrange equation, written for a representative finite element of the multilayered plate, $\Omega_{e} \times h$. The independent variables and corresponding variations are then substituted in view of the assumed layerwise variable description given by Equation (1), along with the following two-dimensional approximations:

$$
\begin{aligned}
& u_{s}^{k}(x, y)=\sum_{j=1}^{n_{u}} u_{j s}^{k} \psi_{u j}(x, y), \sigma_{x z s}{ }^{k}(x, y)=\sum_{j=1}^{n_{\sigma_{x z}}} \sigma_{x z j s}{ }^{k} \psi_{\sigma_{x z} j}(x, y) \\
& v_{s}^{k}(x, y)=\sum_{j=1}^{n_{v}} v_{j s}^{k} \psi_{v j}(x, y), \sigma_{y z s}{ }^{k}(x, y)=\sum_{j=1}^{n_{\sigma_{y z}}} \sigma_{y z}{ }^{k} \psi_{j s} \psi_{\sigma_{y z} j}(x, y)
\end{aligned}
$$




$$
\begin{gathered}
w_{s}^{k}(x, y)=\sum_{j=1}^{n_{w}} w_{j s}^{k} \psi_{w_{j}}(x, y), \sigma_{z z s}{ }^{k}(x, y)=\sum_{j=1}^{n_{\sigma_{z z}}} \sigma_{z z j s}{ }^{k} \psi_{\sigma_{z z} j}(x, y) \\
\varepsilon_{x x s}{ }^{k}(x, y)=\sum_{j=1}^{n_{\varepsilon_{x x}}} \varepsilon_{x x}{ }^{k}{ }_{j s} \psi_{\varepsilon_{x x} j}(x, y), \varepsilon_{y y_{s}}{ }^{k}(x, y)=\sum_{j=1}^{n_{\varepsilon_{y y}}} \varepsilon_{y y j s}{ }^{k} \psi_{\varepsilon_{y y} j}(x, y) \\
\gamma_{x y}{ }^{k}(x, y)=\sum_{j=1}^{n_{\gamma_{x y}}} \gamma_{x y}{ }^{k}{ }_{j s} \psi_{\gamma_{x y j} j}(x, y)
\end{gathered}
$$

The functions $\psi_{u j}(x, y), \ldots, \psi_{\gamma_{x y}}(x, y)$ are two-dimensional high-order $C^{0}$ nodal basis functions and $n_{u}, \ldots, n_{\gamma_{x y}}$ the number of nodes in the in-plane finite element. For a $p$-order in-plane rectangular element, the number of nodes is set $n=(p+1)^{2}$. As a result, the variables nodal values $u_{j s}^{k}, \ldots, \gamma_{x y}{ }_{j s}$ correspond to the $j$-in-plane point location at the $s$-plane of the $k$-layer.

Since earlier works on least-squares formulations [21-24] show high-order basis functions as best to truly minimize the least-squares functional, the two-dimensional basis functions adopted are $C^{0}$ interpolant polynomials of Gauss-Lobatto-Legendre quadrature points, which are most suitable for high-order expansions [25]. The tensor product property is preserved, meaning that two-dimensional basis functions are efficiently derived from one-dimensional basis functions. In fact, the assumed expansions in the thickness direction of each $k$-layer also use these one-dimensional basis functions. For clearness, one- and two-dimensional basis functions are denoted as $\phi$ and $\psi$, respectively. Specifically, the one-dimensional $C^{0}$ basis functions of order $p=m-1$ are written using the $p$-order Legendre polynomial $P_{m-1}$, as follows:

$$
\phi_{i}(\xi)=-\frac{\left(1-\xi^{2}\right) P_{m-1}^{\prime}(\xi)}{m(m-1) P_{m-1}\left(\xi_{i}\right)\left(\xi-\xi_{i}\right)}
$$

where $\xi_{i}$ are the $m$ nodes coordinates given by the roots of $\left(1-\xi^{2}\right) P_{m-1}^{\prime}(\xi)=0$, in the interval $[-1,1]$, matching Gauss-Lobatto-Legendre $m$ quadrature points.

Considering the aforementioned substitutions of Equations (1) and (12) into the Euler-Lagrange equation derived from the functional in Equation (11), the layerwise mixed least-squares finite element model is obtained. It should be noted that the sum in Equation (11), to take into account the contribution of each layer in the element $\Omega_{e} \times h$, means in fact the assembly from layer to multilayer. Hence, given the number of nodes through the layer thickness $m$, the multilayer plate total number of nodes through the thickness becomes $(m-1) \times N+1$. To better distinguish between layer and multilayer, the dummy index $s$ used at the layer level becomes $\bar{s}$ at multilayer level. Once all substitutions are carried out, the layerwise mixed least-squares model for static analysis of multilayered composite plates, ultimately takes the following matrix form: 


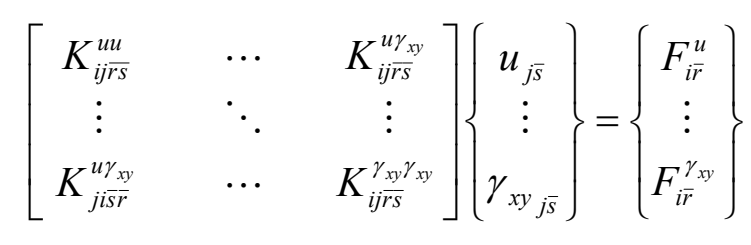

where a Kronecker product is used between the result of the integration in $\Omega_{e}$ and the result of the integration in $h$, as generally specified below:

$$
K_{i j \bar{r} s}^{c d}=K_{i j}^{c d} \otimes K_{\overline{r s}}^{c d}, F_{i \bar{r}}^{c}=F_{i}^{c} \otimes F_{\bar{r}}^{c}
$$

Furthermore, the layerwise mixed least-squares model surely gives a symmetric positive-definite system of linear equations in the end. In fact, symmetry of the stiffness matrix is specified in Equation (14), in view of the transpose property over the Kronecker product, as generally given:

$$
\left(K_{i j \bar{r} s}^{c d}\right)^{t}=\left(K_{i j}^{c d}\right)^{t} \otimes\left(K_{\overline{r s}}^{c d}\right)^{t}=K_{j i}^{c d} \otimes K_{\bar{s} \bar{r}}^{c d}=K_{j i \bar{r} r}^{c d}=K_{i j \bar{r} s}^{d c}
$$

The integral expressions of all submatrices $K_{i j \bar{r} s}^{a b}$ and subvectors $F_{i \bar{r}}^{a}$ in Equation (14) are given in the Report Project [26]. To be clear, it should be underlined that the present model uses full integration in the numerical evaluation of all integrals, since earlier works show that mixed least-squares models are insensitive to shear-locking [21-24]. Also, once all independent variables are solved, the in-plane stresses and transverse strains may be post-computed using Equations (6a) and (6b), respectively.

\subsubsection{Boundary Conditions}

In the static analysis of multilayered composite plates, with a transverse load $q(x, y)$ applied on the plate top surface, boundary conditions on the transverse stresses are generally considered on the top and bottom plate surfaces. Since the present model uses transverse stresses as independent variables, in a layerwise variable description, these boundary conditions can be imposed exactly as follows:

$$
\begin{gathered}
\sigma_{x z b}{ }^{1}=0, \sigma_{y z b}{ }^{1}=0, \sigma_{z z b}{ }^{1}=0 \\
\sigma_{x z t}^{N}=0, \sigma_{y z t}^{N}=0, \sigma_{z z t}^{N}=q(x, y)
\end{gathered}
$$

In addition, boundary conditions adequate to the plate edges support type need to be considered concurrently. According to simply supported, clamped or free support types, boundary conditions on displacements and/or in-plane stresses are generally considered. Displacements boundary conditions can be directly imposed, given that they are independent variables of the present model, but in-plane stresses boundary conditions cannot. Nevertheless, least-squares formulations allow the introduction of additional residuals in the functional, corresponding to boundary conditions imposed 
in a least-squares sense. In this case, Equation (6a) is used to write the in-plane stresses in terms of the model independent variables, so that additional residuals can be included in the least-squares functional. For instance the boundary condition,

$$
\sigma_{x x}{ }^{k}=0, \quad \text { at } x=x_{0}
$$

imposed in a least-squares sense corresponds to the following additional term in the functional $J$ :

$$
\left.\frac{1}{2} \sum_{k=1}^{N} \int_{y_{e}} \int_{h^{k}}\left(\hat{C}_{11}^{k} \varepsilon_{x x}{ }^{k}+\hat{C}_{12}{ }^{k} \varepsilon_{y y}{ }^{k}+\hat{C}_{16}{ }^{k} \gamma_{x y}{ }^{k}+\hat{C}_{13}{ }^{k} \sigma_{z z}{ }^{k}\right)^{2}\right|_{x=x_{0}} d z d y_{e}
$$

Ultimately, the present model static analysis of multilayered composite plates uses boundary conditions on the top and bottom plate surfaces, as given by Equation (17), and boundary conditions for the multilayer plate edges, consistent with simply supported, clamped or free support types, which are imposed exactly as follows:

- Simply Supported

$$
\begin{aligned}
& v^{k}=0, w^{k}=0, \varepsilon_{y y}{ }^{k}=0, \quad \text { at } x=0, a \\
& \sigma_{x x}{ }^{k}=0 \text {, in least-squares sense, at } x=0, a \\
& u^{k}=0, w^{k}=0, \varepsilon_{x x}{ }^{k}=0, \quad \text { at } y=0, b \\
& \sigma_{y y}{ }^{k}=0 \text {, in least-squares sense, at } y=0, b
\end{aligned}
$$

- Clamped

$$
\begin{array}{ll}
u^{k}=0, v^{k}=0, w^{k}=0, \varepsilon_{y y}{ }^{k}=0, & \text { at } x=0, a \\
u^{k}=0, v^{k}=0, w^{k}=0, \varepsilon_{x x}{ }^{k}=0, & \text { at } y=0, b
\end{array}
$$

- Free

$$
\begin{array}{ll}
{\sigma_{x x}{ }^{k}}^{k}=0, \sigma_{x y}{ }^{k}=0, \text { in least-squares sense, } & \text { at } x=0, a \\
\sigma_{y y}{ }^{k}=0,{\sigma_{x y}}^{k}=0, \text { in least-squares sense, } & \text { at } y=0, b
\end{array}
$$

\section{Numerical Examples}

Numerical examples are now presented to demonstrate the predictive capabilities of the developed layerwise mixed least-squares finite element model. The assessment of the present model is mainly based on a thorough and complete comparison with three-dimensional elasticity solutions by Pagano and Hatfield [8, 9]. Actually, the 
methodology described by Pagano [8] to derive exact three-dimensional solutions was implemented by the authors using symbolic computing, so that solutions could be presented with the desired precision, some even not available in literature. Since three-dimensional elasticity solutions are only possible for a few cases, with simple geometries and specific stacking sequences, the numerical examples shown are within that scope. In addition, further assessment of the present model is facilitated by considering some results from other finite element models available in literature, above all, the layerwise mixed weak form model by Carrera as reported in [15].

The numerical examples focus on a benchmark problem of static analysis of the simply supported square multilayered composite plate (0/90/0), with a bi-sinusoidal transverse load of intensity $q_{0}$ applied on the plate top surface. The outer layers total thickness equals the central layer thickness, so the more intuitive form $(0 / 90 / 90 / 0)$ is used henceforth. A range of side-to-thickness ratios is considered, including very thick, moderately thick, thin and very thin plates, $a / h=2,4,10,20,50,100,500$. The material properties used for all layers are the same, as follows:

$$
\begin{aligned}
E_{1} / E_{2} & =25, E_{3}=E_{2}, G_{12}=G_{13}=0.5 E_{2} \\
G_{23} & =0.2 E_{2}, v_{12}=v_{13}=v_{23}=0.25
\end{aligned}
$$

To facilitate assessment, all results are in a non-dimensionalized form, as shown:

$$
\begin{gathered}
\left(\bar{\sigma}_{x x}, \bar{\sigma}_{y y}, \bar{\sigma}_{x y}\right)=\frac{1}{q_{0}(a / h)^{2}}\left(\sigma_{x x}, \sigma_{y y}, \sigma_{x y}\right) \\
\left(\bar{\sigma}_{x z}, \bar{\sigma}_{y z}\right)=\frac{1}{q_{0}(a / h)}\left(\sigma_{x z}, \sigma_{y x}\right), \bar{\sigma}_{z z}=\frac{1}{q_{0}} \sigma_{z z} \\
(\bar{u}, \bar{v})=\frac{E_{2}}{q_{0} h(a / h)^{3}}(u, v), \bar{w}=\frac{100 E_{2}}{q_{0} h(a / h)^{4}} w
\end{gathered}
$$

The following results of the layerwise mixed least-squares model used at all times a mesh of $3 \times 3$ in-plane elements of 4th-order basis functions $\psi(x, y)$ and 3 layers of 3 rd-order basis functions $\phi(z)$. This mesh seemed sufficient to achieve comparable results with the three-dimensional solutions as well as other finite element results available in literature. However, in truth, it would be interesting to refine this mesh even further, layerwise, whether by adding more (numerical) layers or by increasing the $z$-expansion order. This is intended for the near future, but to this date those results are not yet available. This is due to the increase in memory requirements, which involved processing in a different platform.

\subsection{Composite Plate (0/90/90/0) Static Analysis}

An initial overview of the present model accuracy compared to other finite element models, in the prediction of the transverse displacement, is summarized in Table 1, 
considering $a / h=4,10,20,100$, along with the exact three-dimensional solutions by Pagano and Hatfield $[8,9]$. Specifically, the results presented by other finite element models are available in the complete paper by Carrera [15], dedicated to numerical evaluations of multilayered plate elements. Among several finite element models developed by Carrera for evaluation, only the best is included here for comparison, which corresponds to a layerwise mixed weak form model of 4th-order $z$-expansion in the layer thickness, henceforth denoted as Carrera model. The other finite element models also included are designated by the same acronyms as in Carrera [15], to be clear. Those results are in fact from Reddy (R-H) [27], Di and Ramm (D\&R) [28] and Liew, Han and Xiao (LH\&X) [29].

\begin{tabular}{ccccc}
\hline Model & $a / h=4$ & $a / h=10$ & $a / h=20$ & $a / h=100$ \\
\hline 3D & 1.93672 & 0.73698 & 0.51295 & 0.43460 \\
present & 1.93317 & 0.73662 & 0.51291 & 0.43459 \\
Carrera & 1.9374 & 0.7376 & 0.5133 & 0.4348 \\
R-H & 1.8937 & 0.7147 & 0.5060 & 0.4343 \\
D\&R & 1.9530 & 0.7377 & 0.5122 & 0.4333 \\
LH\&X & 1.7095 & 0.6627 & 0.4912 & 0.4337 \\
\hline
\end{tabular}

Table 1: Comparison of transverse displacement $\bar{w}\left(\frac{a}{2}, \frac{a}{2}, 0\right)$ by the present model with other available models for the composite plate $(0 / 90 / 90 / 0)$.

In addition, a complete assessment of the present layerwise mixed least-squares model results for the static analysis of the multilayered composite plate $(0 / 90 / 90 / 0)$, considering $a / h=2,4,10,20,50,100,500$, is given in Table 2 by comparison with three-dimensional solutions $[8,9]$. Carrera model results are also included to better contrast this other layerwise mixed model based on a weak formulation, instead.

\begin{tabular}{ccccccc}
\hline Model & $\begin{array}{c}\bar{\sigma}_{x x} \\
\left(\frac{a}{2}, \frac{a}{2}, \pm \frac{h}{2}\right)\end{array}$ & $\begin{array}{c}\bar{\sigma}_{y y}{ }^{k=2} \\
\left(\frac{a}{2}, \frac{a}{2}, \pm \frac{h}{4}\right)\end{array}$ & $\begin{array}{c}\bar{\sigma}_{x y} \\
\left(0,0, \pm \frac{h}{2}\right)\end{array}$ & $\begin{array}{c}\bar{\sigma}_{x z} \\
\left(0, \frac{a}{2}, 0\right)\end{array}$ & $\begin{array}{c}\bar{\sigma}_{y z} \\
\left(\frac{a}{2}, 0,0\right)\end{array}$ & $\begin{array}{c}\bar{w} \\
\left(\frac{a}{2}, \frac{a}{2}, 0\right)\end{array}$ \\
\hline a/h=2 & & & & & & \\
3D & 1.38841 & 0.83508 & -0.08630 & 0.15300 & 0.29458 & 5.07450 \\
& -0.91165 & -0.79465 & 0.06732 & & & \\
present & 1.11045 & 0.52324 & -0.08630 & 0.15396 & 0.31550 & 5.07759 \\
& -0.74610 & -0.58870 & 0.06697 & & & \\
Carrera & 1.4405 & 0.8478 & -0.0911 & 0.1601 & 0.3105 & 5.0800 \\
a/h=4 & -0.9444 & -0.8116 & 0.0710 & & & \\
3D & 0.72026 & 0.66255 & -0.04666 & 0.21933 & 0.29152 & 1.93672 \\
present & -0.68434 & -0.66551 & 0.04581 & & & \\
Carrera & -0.67552 & 0.58800 & -0.04648 & 0.21944 & 0.29978 & 1.93317 \\
& 0.7456 & -0.60045 & 0.04563 & & & \\
& -0.7093 & -0.6897 & -0.0493 & 0.2294 & 0.3148 & 1.9374 \\
\hline \multicolumn{7}{c}{ Continued }
\end{tabular}




\begin{tabular}{|c|c|c|c|c|c|c|}
\hline Model & $\begin{array}{c}\bar{\sigma}_{x x} \\
\left(\frac{a}{2}, \frac{a}{2}, \pm \frac{h}{2}\right)\end{array}$ & $\begin{array}{c}\bar{\sigma}_{y y}{ }^{k=2} \\
\left(\frac{a}{2}, \frac{a}{2}, \pm \frac{h}{4}\right)\end{array}$ & $\begin{array}{c}\bar{\sigma}_{x y} \\
\left(0,0, \pm \frac{h}{2}\right)\end{array}$ & $\begin{array}{c}\bar{\sigma}_{x z} \\
\left(0, \frac{a}{2}, 0\right)\end{array}$ & $\begin{array}{c}\bar{\sigma}_{y z} \\
\left(\frac{a}{2}, 0,0\right)\end{array}$ & $\begin{array}{c}\bar{w} \\
\left(\frac{a}{2}, \frac{a}{2}, 0\right)\end{array}$ \\
\hline \multicolumn{7}{|l|}{$a / h=10$} \\
\hline \multirow[t]{2}{*}{$3 \mathrm{D}$} & 0.55861 & 0.40095 & -0.02750 & 0.30137 & 0.19595 & 0.73698 \\
\hline & -0.55909 & -0.40257 & 0.02764 & & & \\
\hline \multirow[t]{2}{*}{ present } & 0.55501 & 0.39398 & -0.02766 & 0.30135 & 0.19721 & 0.73662 \\
\hline & -0.55552 & -0.39576 & 0.02780 & & & \\
\hline \multirow[t]{2}{*}{ Carrera } & 0.5909 & 0.4225 & -0.0286 & 0.3073 & 0.1607 & 0.7376 \\
\hline & -05915 & -0.4244 & 0.0287 & & & \\
\hline \multicolumn{7}{|l|}{$a / h=20$} \\
\hline \multirow[t]{2}{*}{$3 \mathrm{D}$} & 0.54282 & 0.30835 & -0.02302 & 0.32816 & 0.15562 & 0.51295 \\
\hline & -0.54320 & -0.30880 & 0.02307 & & & \\
\hline \multirow[t]{2}{*}{ present } & 0.54239 & 0.30720 & -0.02318 & 0.32819 & 0.15580 & 0.51291 \\
\hline & -0.54278 & -0.30766 & 0.02323 & & & \\
\hline \multirow[t]{2}{*}{ Carrera } & 0.5732 & 0.3239 & -0.0239 & 0.3592 & 0.1697 & 0.5133 \\
\hline & -0.5741 & -0.3247 & 0.0240 & & & \\
\hline \multicolumn{7}{|l|}{$a / h=50$} \\
\hline \multirow[t]{2}{*}{$3 \mathrm{D}$} & 0.53931 & 0.27593 & -0.02157 & 0.33740 & 0.14115 & 0.44461 \\
\hline & -0.53938 & -0.27600 & 0.02158 & & & \\
\hline \multirow[t]{2}{*}{ present } & 0.53934 & 0.27590 & -0.02164 & 0.33755 & 0.14056 & 0.44461 \\
\hline & -0.53941 & -0.27597 & 0.02165 & & & \\
\hline \multirow[t]{2}{*}{ Carrera } & 0.5673 & 0.2894 & -0.0225 & 0.3665 & 0.1533 & 0.4449 \\
\hline & -0.5674 & -0.2895 & 0.0225 & & & \\
\hline \multicolumn{7}{|l|}{$a / h=100$} \\
\hline \multirow[t]{2}{*}{ 3D } & 0.53885 & 0.27101 & -0.02135 & 0.33880 & 0.13894 & 0.43460 \\
\hline & -0.53887 & -0.27103 & 0.02136 & & & \\
\hline \multirow[t]{2}{*}{ present } & 0.53891 & 0.27103 & -0.02139 & 0.33909 & 0.13803 & 0.43459 \\
\hline & -0.53893 & -0.27104 & 0.02139 & & & \\
\hline \multirow[t]{2}{*}{ Carrera } & 0.5655 & 0.2841 & -0.0224 & 0.3665 & 0.1505 & 0.4348 \\
\hline & -0.5655 & -0.2841 & 0.0224 & & & \\
\hline \multicolumn{7}{|l|}{$a / h=500$} \\
\hline \multirow[t]{2}{*}{$3 \mathrm{D}$} & 0.53870 & 0.26941 & -0.02128 & 0.33925 & 0.13823 & 0.43138 \\
\hline & -0.53870 & -0.26942 & 0.02128 & & & \\
\hline \multirow[t]{2}{*}{ present } & 0.53573 & 0.26819 & -0.02119 & 0.33792 & 0.13791 & 0.42900 \\
\hline & -0.53573 & -0.26819 & 0.02119 & & & \\
\hline
\end{tabular}

Table 2: Comparison of results by the present model with 3D solutions and Carrera model for the composite plate $(0 / 90 / 90 / 0)$.

The initial comparative study shown in the former Table 1 is mostly significant in demonstrating that both layerwise mixed models, the present and Carrera, are the most accurately efficient models in predicting the transverse displacement for the static analysis of the multilayered composite plate $(0 / 90 / 90 / 0)$, in the entire range of side-to-thickness ratios considered $a / h=4, \ldots, 100$, although all models give in fact rather acceptable results. The more comprehensive study in Table 2 provides a better understanding of the present model predictive capabilities. Basically, examination of 
Table 2 shows that the present layerwise mixed least-squares model is able to attain quite accurate results in very good agreement with the corresponding exact threedimensional solutions. More specifically, for moderately thick to very thin plates $a / h=10, \ldots, 500$, the level of accuracy of the present model in the prediction of all variables is really quite remarkable, in some instances, nearly coincident with threedimensional solutions. This fact also demonstrates that the present layerwise mixed least-squares model, using full integration, appears to be insensitive to shear-locking problems, even when analysing very thin plates, with $a / h=100,500$. For very thick plates $a / h=2,4$, the level of accuracy of the present model in the prediction of most variables is still quite good compared to three-dimensional solutions, apart from the predictions of the in-plane normal stresses, which appear not as accurate. Actually, Carrera model is only able to achieve more accurate results than the present model precisely in the predicted in-plane normal stresses for $a / h=2,4$, and just slightly in the predicted transverse displacement for $a / h=4$. At this point, it is convenient to underline that the present model uses the same mesh for all the different cases of side-to-thickness ratios, namely, $3 \times 3$ in-plane elements of 4 th-order and 3 layers of 3rd-order. Although this mesh proved to be sufficient for moderately thick to very thin plates, reaching very good predictions for all variables, it is understandable that this mesh may need a further refinement, layerwise, in order to be able to attain the same level of accuracy in much thicker plates. In contrast, Carrera model results are obtained using a mesh of $4 \times 4$ in-plane elements of 2 nd-order and a non-specified number of (numerical) layers of 4th-order, according to Carrera [15]. Hence, the two meshes are fairly comparable in-plane, but Carrera model is already more refined layerwise. Therefore, it cannot really be answered (to this date) whether the present model using the same 4th-order $z$-expansion, would be able to achieve (at least) as much accurate predictions as Carrera model in the few aforementioned instances concerning very thick plates. However, it can be ascertained that in all remaining instances the present model is able to attain quite accurate results in very good agreement with three-dimensional solutions, even more than Carrera model, in spite of a less demanding layerwise description. Furthermore, Carrera model results as shown, needed an extensive use of reduced/selective integration techniques to avoid transverse shear-locking, which is clearly stated in Carrera [15], whereas the present layerwise mixed least-squares model requires no such efforts.

To conclude the assessment of the present model predictive capabilities, Figures 2-4 show the predicted distributions through the plate thickness for the variables $\bar{u}$, $\bar{\sigma}_{x x}$ and $\bar{\sigma}_{x z}$, considering thick, moderately thick and thin plates $a / h=4,10,100$, alongside the three-dimensional solutions. These plots combined with the preceding tables provide a fairly detailed description of the composite plate $(0 / 90 / 90 / 0)$ static analysis. Altogether, Figures 2-4 corroborate with the previous discussion.

In all figures, both three-dimensional solutions and the present model predicted distributions are in fact represented using the corresponding symbolic functions. For the layerwise mixed least-squares model this means that the plots are based on the actual one-dimensional $C^{0}$ basis functions in the layer thickness combined with the variables nodal values, as marked. 


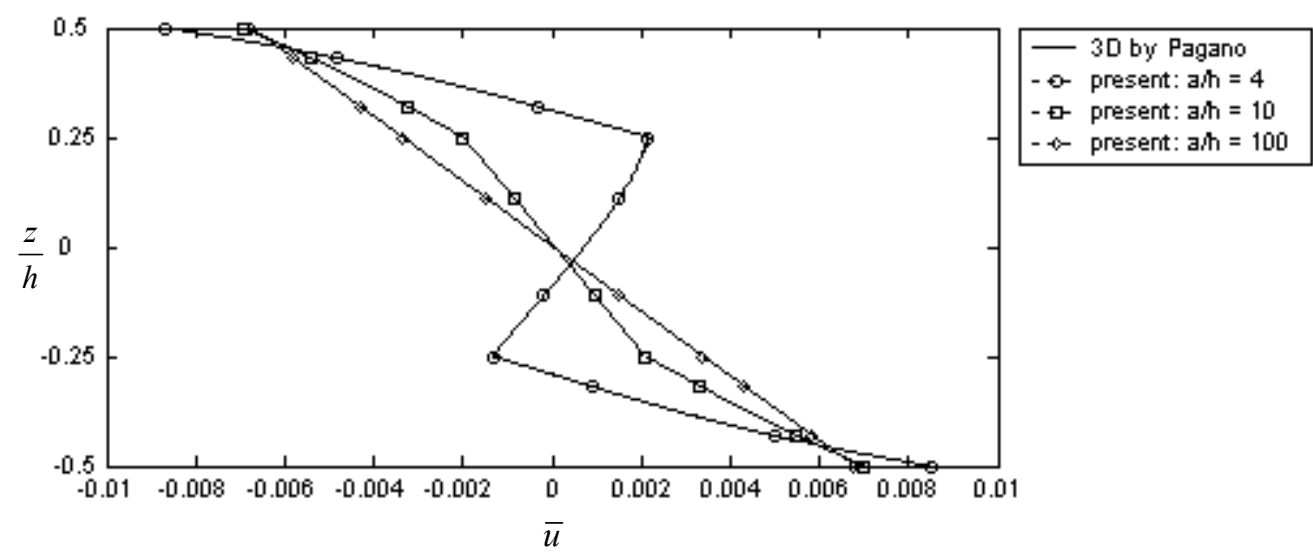

Figure 2: In-plane displacement $\bar{u}\left(0, \frac{a}{2}\right)$ through the thickness for the composite plate $(0 / 90 / 90 / 0)$.

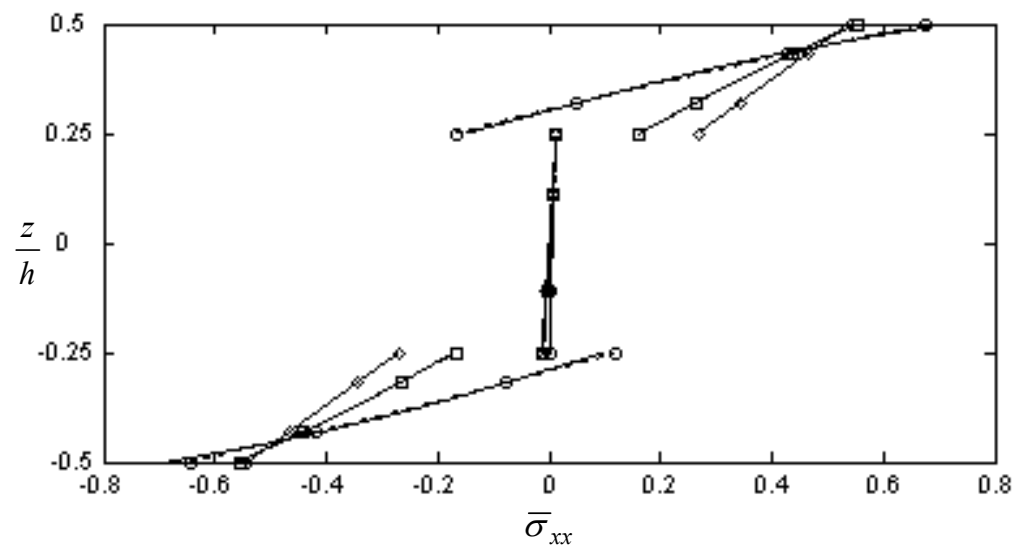
- 3Б by Pagano - to present : ah $=4$
- ๒- Present: $:$ hh $=10$
-+ preserit : an $=1000$

Figure 3: In-plane normal stress $\bar{\sigma}_{x x}\left(\frac{a}{2}, \frac{a}{2}\right)$ through the thickness for the composite plate $(0 / 90 / 90 / 0)$.

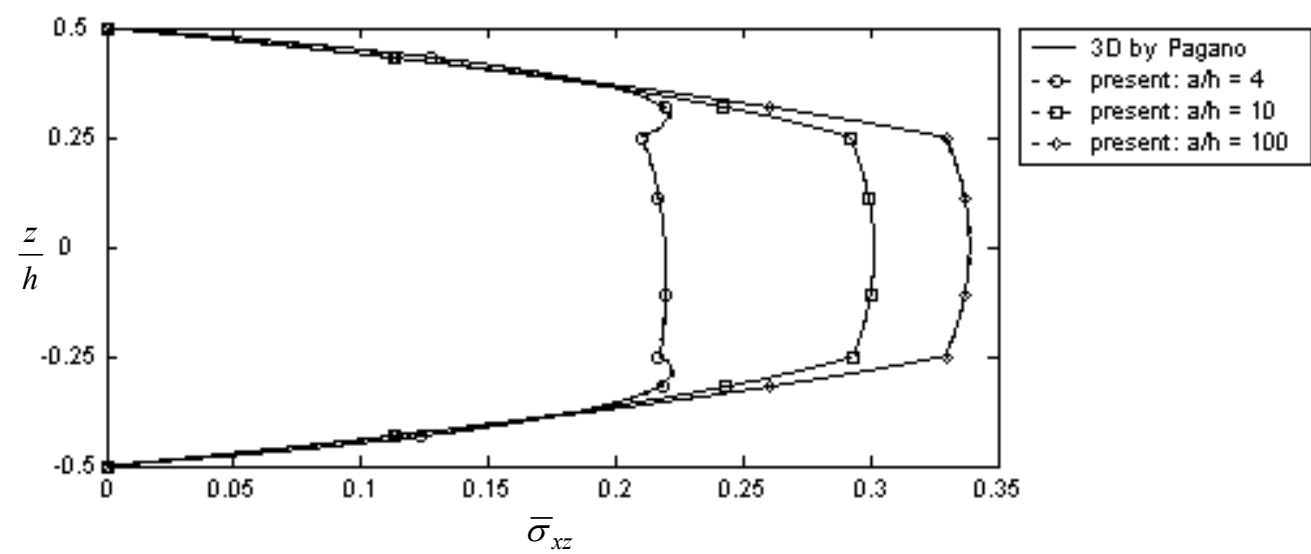

Figure 4: Transverse shear stress $\bar{\sigma}_{x z}\left(0, \frac{a}{2}\right)$ through the thickness for the composite plate $(0 / 90 / 90 / 0)$. 


\section{Concluding Remarks}

A layerwise finite element model is developed in a mixed least-squares formulation for static analysis of multilayered composite plates. An axiomatic type approach is used, with a layerwise variable description of displacements, transverse stresses and in-plane strains, in a truly (partial) mixed formulation. Since multilayered composite structures may exhibit complicating effects introduced by anisotropic behaviour, such as high transverse deformability, zig-zag effects and interlaminar continuity, a layerwise model is really required to be able to accurately capture such effects. In fact, to completely and a priori fulfil $C_{z}^{0}$ requirements, a mixed formulation is also preferred. Furthermore, the mixed least-squares formulation is adopted given that it leads to a variational unconstrained minimization problem, where the finite element approximating spaces can be chosen independently, as opposed to mixed weak form models. Additionally, mixed least-squares models as shown by earlier works [2124] appear to be insensitive to shear-locking, which is a common problem on weak form models, whether displacement-based or mixed.

Numerical examples are then presented to show the predictive capabilities of the developed layerwise mixed least-squares finite element model. The assessment of the present model results is primarily based on a comprehensive comparison with the three-dimensional elasticity solutions by Pagano and Hatfield [8, 9]. Further assessment is also facilitated by comparison with other finite element results, above all, the layerwise mixed weak form model by Carrera [15]. The numerical examples focus on the static analysis of the simply supported square multilayered composite plate $(0 / 90 / 90 / 0)$, under a bi-sinusoidal transverse load, with a range of side-tothickness ratios, including very thick, moderately thick, thin and very thin plates, $a / h=2,4,10,20,50,100,500$. Altogether, it is shown that the present model is able to obtain quite accurate results in very good agreement with the three-dimensional solutions. Specifically, for moderately thick to very thin plates $a / h=10, \ldots, 500$, the present model achieves highly accurate results for all variables, in fact better than Carrera model, even though the $z$-expansion order in the layer thickness is of 3rdorder in the present model and 4th-order in Carrera model. For very thick plates $a / h=2,4$, the present model also reaches rather accurate results, but less so for the in-plane normal stresses that appear not as accurate. This is precisely where Carrera model provides better results, which suggests that the present model might benefit from a further refinement, layerwise, whether by adding more (numerical) layers or by increasing the $z$-expansion order, in order to be able to achieve more accurate results in this case. In effect, such results are expected for the near future. Finally, it is important to underline that unlike Carrera model, the present layerwise mixed least-squares model is shown to be insensitive to shear-locking altogether.

\section{Acknowledgments}

The authors acknowledge the financial support received by FCT/POCTI/FEDER, FCT/POCI(2010)/FEDER, Project PPCDT/EME/56316/2004 and the EU through 
Project FP6-NMP3-CT-2005-013517 "CASSEM". In addition, the first author is grateful for the Grant SFRH/BD/12292/2003 assured by FCT and the last author also thanks the support received from the US Army Research Grant 45508-EG.

\section{References}

[1] E. Carrera, "Theories and finite elements for multilayered, anisotropic, composite plates and shells", Archives of Computational Methods in Engineering, 9(2), 87-140, 2002.

[2] J.N. Reddy, D.H. Robbins Jr, "Theories and computational models for composite laminates", Applied Mechanics Reviews, 47(6), 147-169, 1994.

[3] A.K. Noor, W.S. Burton, "Assessment of shear deformation theories for multilayered composite plates", Applied Mechanics Reviews, 42(1), 1-13, 1989.

[4] J.N. Reddy, "Mechanics of Laminated Composite Plates and Shells - Theory and Analysis", CRC Press, Boca Raton, 2004.

[5] J.N. Reddy, "An Introduction to the Finite Element Method", McGraw-Hill, New York, 2004.

[6] K. Washizu, "Variational Methods in Elasticity and Plasticity", Pergamon Press, New York, 1982.

[7] J.N. Reddy, "Energy Principles and Variational Methods in Applied Mechanics", John Wiley, New York, 2002.

[8] N.J. Pagano, "Exact solutions for rectangular bidirectional composites and sandwich plates", Journal of Composite Materials, 4, 20-34, 1970.

[9] N.J. Pagano, S.J. Hatfield, "Elastic behavior of multilayered bidirectional composites", American Institute of Aeronautics and Astronautics Journal, 10, 931-933, 1972.

[10] E. Carrera, " $C_{z}^{0}$ Requirements - models for the two dimensional analysis of multilayered structures", Composite Structures, 37(3/4), 373-383, 1997.

[11] E. Carrera, "Developments, ideas, and evaluations based upon Reissner's mixed variational theorem in the modeling of multilayered plates and shells", Applied Mechanics Reviews, 54(4), 301-329, 2001.

[12] E. Reissner, "On a certain mixed variational theory and a proposed application", International Journal for Numerical Methods in Engineering, 20, 1366-1368, 1984.

[13] E. Reissner, "On a mixed variational theorem and on a shear deformable plate theory", International Journal for Numerical Methods in Engineering, 23, 193198, 1986.

[14] E. Carrera, L. Demasi, "Classical and advanced multilayered plate elements based upon PVD and RMVT. Part 1: Derivation of finite element matrices", International Journal for Numerical Methods in Engineering, 55, 191-231, 2002.

[15] E. Carrera, L. Demasi, "Classical and advanced multilayered plate elements based upon PVD and RMVT. Part 2: Numerical implementations", International Journal for Numerical Methods in Engineering, 55, 253-291, 
2002.

[16] E. Carrera, "An assessment of mixed and classical theories on global and local response of multilayered orthotropic plates", Composite Structures, 50, 183198, 2000.

[17] E. Carrera, "A priori vs. a posteriori evaluation of transverse stresses in multilayered orthotropic plates", Composite Structures, 48, 245-260, 2000.

[18] E. Carrera, "Evaluation of layerwise mixed theories for laminated plates analysis", American Institute of Aeronautics and Astronautics Journal, 36(5), 830-839, 1998.

[19] R.G. Lage, C.M. Mota Soares, C.A. Mota Soares, J.N. Reddy, "Modelling of piezolaminated plates using layerwise mixed finite elements", Computers and Structures, 82, 1849-1863, 2004.

[20] R.G. Lage, C.M. Mota Soares, C.A. Mota Soares, J.N. Reddy, "Layerwise partial mixed finite element analysis of magneto-electro-elastic plates", Computers and Structures, 82, 1293-1301, 2004.

[21] J.P. Pontaza, J.N. Reddy, "Mixed plate bending elements based on leastsquares formulation", International Journal for Numerical Methods in Engineering, 60, 891-922, 2004.

[22] J.P. Pontaza, "Least-squares variational principles and the finite element method: theory, formulations, and models for solid and fluid mechanics", Finite Elements in Analysis and Design, 41, 703-728, 2005.

[23] F. Moleiro, C.M. Mota Soares, C.A. Mota Soares, J.N. Reddy, "Mixed leastsquares finite element model for the static analysis of laminated composite plates", Computers and Structures, 86, 826-838, 2008.

[24] F. Moleiro, C.M. Mota Soares, C.A. Mota Soares, J.N. Reddy, "Mixed leastsquares finite element models for static and free vibration analysis of laminated composite plates", Computer Methods in Applied Mechanics and Engineering, submitted.

[25] T.C. Warburton, S.J. Sherwin, G.E. Karniadakis, "Basis functions for triangular and quadrilateral high-order elements", SIAM Journal on Scientific Computing, 20(5), 1671-1695, 1999.

[26] F. Moleiro, "Layerwise mixed least-squares finite element model for static analysis of multilayered composite plates: theory and applications", Report Project PPCDT/EME/56136/2004 - IDMEC/IST March 2008.

[27] J.N. Reddy, "A simple higher-order theory for laminated composite plates", Journal of Applied Mechanics, 51, 745-752, 1985.

[28] S. Di, E. Ramm, "Hybrid stress formulation for higher-order theory of laminated shell analysis", Computer Methods in Applied Mechanics and Engineering, 109, 359-376, 1993.

[29] K.M. Liew, B. Han, M. Xiao, "Differential quadrature method for thick symmetric cross-ply laminates with first-order shear flexibility", International Journal of Solids and Structures, 33, 2647-2658, 1996. 